\title{
Some unrestricted Fibonacci and Lucas hyper-complex numbers
}

\author{
Göksal Bilgici and Ahmet DaşDemir
}

\begin{abstract}
A number of studies have investigated the Fibonacci quaternions and octonions that include consecutive terms of the Fibonacci sequence. This paper presents a new generalization of Fibonacci quaternions, octonions and sedenions, where non-consecutive Fibonacci numbers are used. We present the Binet formulas, generating functions and some identities for these new types of hyper-complex numbers.
\end{abstract}

\section{Introduction}

Real number algebras can be converted into hyper-complex number algebras by applying the Cayley-Dickson process (so-called Cayley-Dickson doubling). Using this process, by starting from the real numbers, we successively obtain the well-known complex numbers, quaternions, octonions, sedenions, $2^{N}$-ons. Each algebra is a sub-algebra of all the previous ones. However, increasing the dimension of the algebra results in the loss of some properties, for example, complex numbers do not have the self-conjugacy properties that real algebras have and octonions are non-associative. These lost properties often lead to unexpected results.

The hyper-complex numbers known as $2^{N}$-ons can be regarded as linear combinations of elements from a canonical basis set:

$$
\omega=\sum_{i=0}^{2^{N}-1} w_{i} e_{i}=w_{0} e_{0}+w_{1} e_{1}+\cdots+w_{2^{N}-1} e_{2^{N}-1},
$$

where the $e_{i}$ 's are elements of the basis set and the $w_{i}$ 's are real numbers.

Received November 27, 2018.

2010 Mathematics Subject Classification. 11B39, 11R52.

Key words and phrases. Fibonacci quaternion, Fibonacci octonion, Fibonacci sedenion. https://doi.org/10.12697/ACUTM.2020.24.03

Corresponding author: Göksal Bilgici 
In terms of this basis, the conjugate of $\omega$ is

$$
\omega^{*}=w_{0} e_{0}-\sum_{i=0}^{2^{N}-1} w_{i} e_{i}=w_{0} e_{0}-w_{1} e_{1}-\cdots-w_{2^{N}-1} e_{2^{N}-1}
$$

and the norm of $\omega$ is

$$
N(\omega)=\sum_{i=0}^{2^{N}-1} w_{i}^{2} .
$$

Hyper-complex numbers have been studied by many researchers. In particular, many papers have been devoted to cases such as $N=2$ (quaternions), $N=3$ (octonions) and $N=4$ (sedenions). Table 1 , taken from the paper [3], summarizes the multiplication rules for the bases used by these algebras. For abbreviation, we set $i \equiv e_{i}$ for $i=0, \ldots 15$.

TABLE 1. Multiplication table for $2^{N}$-ons for values of $N$ between 0 and 4

\begin{tabular}{r|rrrrrrrrrrrrrrrr}
$\cdot$ & 0 & 1 & 2 & 3 & 4 & 5 & 6 & 7 & 8 & 9 & 10 & 11 & 12 & 13 & 14 & 15 \\
\hline 0 & 0 & 1 & 2 & 3 & 4 & 5 & 6 & 7 & 8 & 9 & 10 & 11 & 12 & 13 & 14 & 15 \\
1 & 1 & -0 & 3 & -2 & 5 & -4 & -7 & 6 & 9 & -8 & -11 & 10 & -13 & 12 & 15 & -14 \\
2 & 2 & -3 & -0 & 1 & 6 & 7 & -4 & -5 & 10 & 11 & -8 & -9 & -14 & -15 & 12 & 13 \\
3 & 3 & 2 & -1 & -0 & 7 & -6 & 5 & -4 & 11 & -10 & 9 & -8 & -15 & 14 & -13 & 12 \\
4 & 4 & -5 & -6 & -7 & -0 & 1 & 2 & 3 & 12 & 13 & 14 & 15 & -8 & -9 & -10 & -11 \\
5 & 5 & 4 & -7 & 6 & -1 & -0 & -3 & 2 & 13 & -12 & 15 & -14 & 9 & -8 & 11 & -10 \\
6 & 6 & 7 & 4 & -5 & -2 & 3 & -0 & -1 & 14 & -15 & -12 & 13 & 10 & -11 & -8 & 9 \\
7 & 7 & -6 & 5 & 4 & -3 & -2 & 1 & -0 & 15 & 14 & -13 & -12 & 11 & 10 & -9 & -8 \\
8 & 8 & -9 & -10 & -11 & -12 & -13 & -14 & -15 & -0 & 1 & 2 & 3 & 4 & 5 & 6 & 7 \\
9 & 9 & 8 & -11 & 10 & -13 & 12 & 15 & -14 & -1 & -0 & -3 & 2 & -5 & 4 & 7 & -6 \\
10 & 10 & 11 & 8 & -9 & -14 & -15 & 12 & 13 & -2 & 3 & -0 & -1 & -6 & -7 & 4 & 5 \\
11 & 11 & -10 & 9 & 8 & -15 & 14 & -13 & 12 & -3 & -2 & 1 & -0 & -7 & 6 & -5 & 4 \\
12 & 12 & 13 & 14 & 15 & 8 & -9 & -10 & -11 & -4 & 5 & 6 & 7 & -0 & -1 & -2 & -3 \\
13 & 13 & -12 & 15 & -14 & 9 & 8 & 11 & -10 & -5 & -4 & 7 & -6 & 1 & -0 & 3 & -2 \\
14 & 14 & -15 & -12 & 13 & 10 & -11 & 8 & 9 & -6 & -7 & -4 & 5 & 2 & -3 & -0 & 1 \\
15 & 15 & 14 & -13 & -12 & 11 & 10 & -9 & 8 & -7 & 6 & -5 & -4 & 3 & 2 & -1 & -0
\end{tabular}

Since the inception of hyper-complex number theory, many authors have investigated quaternions, octonions, and sedenions whose coefficients have been taken from special integer sequences, such as the Fibonacci and Lucas sequences, in different ways. The Fibonacci numbers $\left\{F_{n}\right\}_{n=0}^{\infty}$ are recursively defined via the relation $F_{n+1}=F_{n}+F_{n-1}$ with initial terms $F_{0}=0$ and $F_{1}=1$, and the Lucas numbers $\left\{L_{n}\right\}_{n=0}^{\infty}$ are defined by the same recursive equation but with initial terms $L_{0}=2$ and $L_{1}=1$. Horadam [6] defined Fibonacci quaternions as

$$
Q_{n}:=F_{n}+i F_{n+1}+j F_{n+2}+k F_{n+3} .
$$

Iyer [7] gave a similar definition for Lucas quaternions via the relation

$$
T_{n}:=L_{n}+i L_{n+1}+j L_{n+2}+k L_{n+3},
$$


as well as presenting many properties of Fibonacci quaternions. Halici [5] investigated Binet's formulas and generating functions for Fibonacci and Lucas quaternions. Fibonacci and Lucas quaternions have been extensively studied, and many generalizations have been considered. For instance, Swamy [10] considered the new quaternion $R_{n}$ defined as

$$
R_{n}:=M_{n}+i M_{n+1}+j M_{n+2}+k M_{n+3},
$$

where $M_{n+1}=M_{n}+M_{n-1}$, with initial terms $M_{0}=s$ and $M_{1}=r$. Flaut and Shpakivskyi [4] gave certain properties of generalized Fibonacci quaternions and Fibonacci-Narayana quaternions. Akyigit et al. [1] introduced the Fibonacci generalized quaternions and described many of their properties. Kecillioglu and Akkus [8] extended the definitions of Horadam [6] and Iyer [7] to Fibonacci and Lucas octonions as follows:

$$
Q_{n}:=\sum_{i=0}^{7} F_{i} e_{i} \text { and } T_{n}:=\sum_{i=0}^{7} L_{i} e_{i} .
$$

They have also authors presented Binet's formulas and some other identities for these types of octonions.

In addition, Bilgici et al. mentioned Fibonacci and Lucas sedenions in their study [2].

Note that all these authors introduced Fibonacci and Lucas hyper-complex numbers and their generalizations into consecutive basis coefficients $\left\{e_{0}, e_{1}, \ldots, e_{2^{N}-1}\right\}$ as in Horadam [6] and Iyer [7]. In this paper, however, we present a new perspective on Fibonacci and Lucas quaternions, octonions and sedenions. We introduce unrestricted Fibonacci and Lucas $2^{N}$-ons and present several properties of these hyper-complex numbers.

Throughout the paper, we take $\vec{c}=\left(c_{0}, c_{1}, \ldots, c_{2^{N}-1}\right)$ where $c_{0}=0$ and $c_{1}, c_{2}, \ldots, c_{2^{N}-1}$ are integers.

Definition 1. The $r$ th unrestricted Fibonacci and Lucas $2^{N}$-ons are defined, respectively, by the relations

$$
\mathcal{F}_{N, r}^{\vec{c}}:=\sum_{i=0}^{2^{N}-1} F_{r+c_{i}} e_{i} \text { and } \mathcal{L}_{N, r}^{\vec{c}}:=\sum_{i=0}^{2^{N}-1} L_{r+c_{i}} e_{i} .
$$

From the defining recurrence relations for Fibonacci and Lucas numbers, we can see that unrestricted Fibonacci and Lucas $2^{N}$-ons satisfy the recurrence relations

and

$$
\mathcal{F}_{N, r}^{\vec{c}}=\mathcal{F}_{N, r-1}^{\vec{c}}+\mathcal{F}_{N, r-2}^{\vec{c}}
$$

respectively.

$$
\mathcal{L}_{N, r}^{\vec{c}}=\mathcal{L}_{N, r-1}^{\vec{c}}+\mathcal{L}_{N, r-2}^{\vec{c}},
$$


Depending on the choice of $N$ and $\vec{c}$, some particular examples of our newly defined $2^{N}$-ons are as follows.

- For $N=0$ and $\vec{c}=(0)$, the original Fibonacci and Lucas (integer) numbers are obtained.

- For $N=1$ and $\vec{c}=(0,1)$, Fibonacci and Lucas complex numbers are obtained.

- For $N=2$ and $\vec{c}=(0,1,2,3)$, Fibonacci and Lucas quaternions are obtained.

- For $N=3$ and $\vec{c}=(0,1, \ldots, 7)$, Fibonacci and Lucas octonions are obtained.

- For $N=4$ and $\vec{c}=(0,1, \ldots, 15)$, Fibonacci and Lucas sedenions are obtained.

We now give an example to clarify these ideas. Consider the Fibonacci quaternion $F_{5} e_{0}+F_{-6} e_{1}+F_{22} e_{2}+F_{0} e_{3}$. The most important coefficient for us is the coefficient of $e_{0}$, i.e., the real part. We write this Fibonacci quaternion as $\mathcal{F}_{2,5}^{(0,-11,17,-5)}$.

The identities $F_{-n}=(-1)^{n+1} F_{n}$ and $L_{-n}=(-1)^{n} L_{n}$ also allow us to give unrestricted Fibonacci and Lucas quaternions with negative indices $r$ as follows:

$$
\mathcal{F}_{N,-r}^{\vec{c}}:=(-1)^{r+1}\left[\sum_{i=0}^{2^{N}-1}(-1)^{c_{i}} F_{r-c_{i}} e_{i}\right]
$$

and

$$
\mathcal{L}_{N,-r}^{\vec{c}}:=(-1)^{r}\left[\sum_{i=0}^{2^{N}-1}(-1)^{c_{i}} L_{r-c_{i}} e_{i}\right] .
$$

\section{Results}

The first result is the Binet's formulas for our unrestricted Fibonacci and Lucas $2^{N}$-ons which are given by the following theorem.

Theorem 1. For any non negative integer $r$, the unrestricted Fibonacci and Lucas $2^{N}$-ons are, respectively,

$$
\mathcal{F}_{N, r}^{\vec{c}}=\frac{\breve{\alpha} \alpha^{r}-\breve{\beta} \beta^{r}}{\alpha-\beta}
$$

and

$$
\mathcal{L}_{N, r}^{\vec{c}}=\breve{\alpha} \alpha^{r}+\breve{\beta} \beta^{r}
$$

where

$$
\breve{\alpha}=\sum_{i=0}^{2^{N}-1} \alpha^{c_{i}} e_{i} \text { and } \breve{\beta}=\sum_{i=0}^{2^{N}-1} \beta^{c_{i}} e_{i} .
$$


Proof. From the definition of unrestricted Fibonacci $2^{N}$-ons and Binet's formula for the Fibonacci numbers we have

$$
\begin{aligned}
\mathcal{F}_{N, r}^{\vec{c}}= & F_{r}+F_{n+c_{1}} e_{1}+\cdots+F_{n+c_{2}{ }_{-1}} e_{2^{N}-1} \\
= & \frac{1}{\alpha-\beta}\left(\alpha^{r}-\beta^{r}+\left(\alpha^{r+c_{1}}-\beta^{r+c_{1}}\right) e_{1}+\left(\alpha^{r+c_{2}}-\beta^{r+c_{2}}\right) e_{2}\right. \\
& \left.+\cdots+\left(\alpha^{r+c_{2}{ }^{N}-1}-\beta^{r+c_{2} N_{-1}}\right) e_{2^{N}-1}\right) \\
= & \frac{1}{\alpha-\beta}\left(\alpha^{r}\left(1+\alpha^{c_{1}} e_{1}+\alpha^{c_{2}} e_{2}+\cdots+\alpha^{c_{2}{ }^{N}-1} e_{2^{N}-1}\right)\right. \\
& \left.-\beta^{r}\left(1+\beta^{c_{1}} e_{1}+\beta^{c_{2}} e_{2}+\cdots+\beta^{c_{2}{ }^{N}} e_{2^{N}-1}\right)\right) .
\end{aligned}
$$

We can obtain (2) from the last equation, and (3) can be proved similarly.

For $i, j \in\left\{1,2, \ldots,\left(2^{N-1}-1\right)\right\}$ and $i \neq j$, we define

$$
S_{i}:=\left\{(j, k) \mid e_{i} e_{j}=e_{k}, 1 \leq j, k \leq\left(2^{N-1}-1\right), i \neq j, i \neq k \text { and } j \neq k\right\} .
$$

For example, for $N=5$, we have

$$
S_{12}=\{(1,13),(2,14),(3,15),(4,8),(9,5),(10,6),(11,7)\} .
$$

We now need to present the following properties of $\breve{\alpha}$ and $\breve{\beta}$ since they will play key roles in the proofs of subsequent theorems.

Lemma 1. For $N \in\{0,1,2,3,4\}$, we have

$$
\breve{\alpha} \breve{\beta}=A_{N}^{\vec{c}}+\sqrt{5} B_{N}^{\vec{c}}
$$

and

where

$$
\breve{\beta} \breve{\alpha}=A_{N}^{\vec{c}}-\sqrt{5} B_{N}^{\vec{c}}
$$

$$
A_{N}^{\vec{c}}=\mathcal{L}_{N, 0}^{\vec{c}}-\sum_{i=0}^{2^{N}-1}(-1)^{c_{i}} \text { and } B_{N}^{\vec{c}}=\sum_{i=1}^{2^{N-1}-1} e_{i} \sum_{(j, k) \in S_{i}}(-1)^{c_{k}} F_{c_{j}-c_{k}} .
$$

Proof. We easily see that

$$
A_{0}^{\vec{c}}=1, \quad A_{1}^{\vec{c}}=\mathcal{L}_{1,0}^{c_{1}}+(-1)^{c_{1}}-1, \quad B_{0}^{\vec{c}}=B_{1}^{\vec{c}}=0 .
$$

For $N=2$, from the definitions of $\breve{\alpha}$ and $\breve{\beta}$ we have

$$
\begin{aligned}
\breve{\alpha} \breve{\beta}= & \left(1+\alpha^{c_{1}} e_{1}+\alpha^{c_{2}} e_{2}+\alpha^{c_{3}} e_{3}\right)\left(1+\beta^{c_{1}} e_{1}+\beta^{c_{2}} e_{2}+\beta^{c_{3}} e_{3}\right) \\
= & 1-(\alpha \beta)^{c_{1}}-(\alpha \beta)^{c_{2}}-(\alpha \beta)^{c_{3}}+\left(\alpha^{c_{1}}+\beta^{c_{1}}+\alpha^{c_{2}} \beta^{c_{3}}-\alpha^{c_{3}} \beta^{c_{2}}\right) e_{1} \\
& +\left(\alpha^{c_{2}}+\beta^{c_{2}}+\alpha^{c_{3}} \beta^{c_{1}}-\alpha^{c_{1}} \beta^{c_{3}}\right) e_{2}+\left(\alpha^{c_{3}}+\beta^{c_{3}}+\alpha^{c_{1}} \beta^{c_{2}}-\alpha^{c_{2}} \beta^{c_{1}}\right) e_{3} \\
= & 1+(-1)^{c_{1}+1}+(-1)^{c_{2}+1}+(-1)^{c_{3}+1}+\left(L_{c_{1}}+(-1)^{c_{3}} \sqrt{5} F_{c_{2}-c_{3}}\right) e_{1} \\
& +\left(L_{c_{2}}+(-1)^{c_{1}} \sqrt{5} F_{c_{3}-c_{1}}\right) e_{2}+\left(L_{c_{3}}+(-1)^{c_{2}} \sqrt{5} F_{c_{1}-c_{2}}\right) e_{3} .
\end{aligned}
$$


Applying the identity $F_{-r}=(-1)^{r+1} F_{r}$ and Binet's formulas for the Fibonacci and Lucas numbers yield

$$
A_{2}^{\vec{c}}=\mathcal{L}_{2,0}^{\vec{c}}+(-1)^{c_{1}+1}+(-1)^{c_{2}+1}+(-1)^{c_{3}+1}-1
$$

and

$$
B_{2}^{\vec{c}}=(-1)^{c_{3}} F_{c_{2}-c_{3}} e_{1}+(-1)^{c_{1}} F_{c_{3}-c_{1}} e_{2}+(-1)^{c_{2}} F_{c_{1}-c_{2}} e_{3} .
$$

For $N=3$, we have

$$
A_{3}^{\vec{c}}=\mathcal{L}_{3,0}^{\vec{c}}+(-1)^{c_{1}+1}+(-1)^{c_{2}+1}+\cdots+(-1)^{c_{7}+1}-1
$$

and

$$
\begin{aligned}
B_{3}^{\vec{c}}= & {\left[(-1)^{c_{3}} F_{c_{2}-c_{3}}+(-1)^{c_{5}} F_{c_{4}-c_{5}}+(-1)^{c_{6}} F_{c_{7}-c_{6}}\right] e_{1} } \\
& +\left[(-1)^{c_{1}} F_{c_{3}-c_{1}}+(-1)^{c_{6}} F_{c_{4}-c_{6}}+(-1)^{c_{7}} F_{c_{5}-c_{7}}\right] e_{2} \\
& +\left[(-1)^{c_{2}} F_{c_{1}-c_{2}}+(-1)^{c_{5}} F_{c_{6}-c_{5}}+(-1)^{c_{7}} F_{c_{4}-c_{7}}\right] e_{3} \\
& +\left[(-1)^{c_{1}} F_{c_{5}-c_{1}}+(-1)^{c_{2}} F_{c_{6}-c_{2}}+(-1)^{c_{3}} F_{c_{7}-c_{3}}\right] e_{4} \\
& +\left[(-1)^{c_{2}} F_{c_{7}-c_{2}}+(-1)^{c_{4}} F_{c_{1}-c_{4}}+(-1)^{c_{6}} F_{c_{3}-c_{6}}\right] e_{5} \\
& +\left[(-1)^{c_{3}} F_{c_{5}-c_{3}}+(-1)^{c_{4}} F_{c_{2}-c_{4}}+(-1)^{c_{7}} F_{c_{1}-c_{7}}\right] e_{6} \\
& +\left[(-1)^{c_{1}} F_{c_{6}-c_{1}}+(-1)^{c_{4}} F_{c_{3}-c_{4}}+(-1)^{c_{5}} F_{c_{2}-c_{5}}\right] e_{7} .
\end{aligned}
$$

For the case $N=4$, we calculate

$$
A_{4}^{\vec{c}}=\mathcal{L}_{4,0}^{\vec{c}}+(-1)^{c_{1}+1}+(-1)^{c_{2}+1}+\cdots+(-1)^{c_{15}+1}-1 .
$$

Each coefficient of $e_{i}$ in $B_{4}^{\vec{c}}$ has seven terms. So, it would be too tedious to write $B_{4}^{\vec{c}}$ in open form. For example, we give only coefficient of $e_{12}$. From the definition of the set $S_{12}$, the possible choices of $(j, k)$ are $(1,13),(2,14)$, $(3,15),(4,8),(9,5),(10,6)$ and $(11,7)$. Thus the coefficient of $e_{12}$ is

$$
\begin{aligned}
& (-1)^{c_{13}} F_{c_{1}-c_{13}}+(-1)^{c_{14}} F_{c_{2}-c_{14}}+(-1)^{c_{15}} F_{c_{3}-c_{15}}+(-1)^{c_{8}} F_{c_{4}-c_{8}} \\
& \quad+(-1)^{c_{5}} F_{c_{9}-c_{5}}+(-1)^{c_{6}} F_{c_{10}-c_{6}}+(-1)^{c_{7}} F_{c_{11}-c_{7}} .
\end{aligned}
$$

The other coefficient of $e_{i}$ in $B_{4}^{\vec{c}}$ can be obtained similarly.

Using Lemma 1, we can obtain the useful equation

$$
\breve{\alpha} \breve{\beta}+\breve{\beta} \breve{\alpha}=2 A_{N}^{\vec{c}} \text {. }
$$

The following theorem gives the generating functions.

Theorem 2. The generating functions for unrestricted Fibonacci and Lucas $2^{N}$-ons are

$$
\sum_{i=0}^{\infty} \mathcal{F}_{N, i}^{\vec{c}}=\frac{\mathcal{F}_{N, 0}^{\vec{c}}+x \mathcal{F}_{N,-1}^{\vec{c}}}{1-x-x^{2}}
$$

and

$$
\sum_{i=0}^{\infty} \mathcal{L}_{N, i}^{\vec{c}}=\frac{\mathcal{L}_{N, 0}^{\vec{c}}+x \mathcal{L}_{N,-1}^{\vec{c}}}{1-x-x^{2}}
$$


Proof. Define $F(x)=\sum_{i=0}^{\infty} \mathcal{F}_{N, i}^{\vec{c}} x^{i}$. Expanding the first two terms of $F(x)$, we obtain

$$
F(x)=\mathcal{F}_{N, 0}^{\vec{c}}+\mathcal{F}_{N, 1}^{\vec{c}} x+\sum_{i=2}^{\infty} \mathcal{F}_{N, i}^{\vec{c}} x^{i} .
$$

Multiplying both sides of this equation by $-x$ and $-x^{2}$ yields

$$
-x F(x)=-\mathcal{F}_{N, 0}^{\vec{c}} x-\sum_{i=2}^{\infty} \mathcal{F}_{N, i-1}^{\vec{c}} x^{i}
$$

and

$$
-x^{2} F(x)=-\sum_{i=2}^{\infty} \mathcal{F}_{N, i-2}^{\vec{c}} x^{i},
$$

respectively. Adding the equations (9), (10) and (11), and applying the recurrence relation $(1)$, we see that

$$
f(x)=\frac{\mathcal{F}_{N, 0}^{\vec{c}}+\left(\mathcal{F}_{N, 1}^{\vec{c}}-\mathcal{F}_{N, 0}^{\vec{c}}\right) x}{1-x-x^{2}}
$$

Using the identity $\mathcal{F}_{N,-1}^{\vec{c}}=\mathcal{F}_{N, 1}^{\vec{c}}-\mathcal{F}_{N, 0}^{\vec{c}}$, we get (7). The equality (8) can be obtained in similar way.

\section{Some identities}

By using Binet's formulas and properties of the Fibonacci and Lucas numbers, we now present several identities for unrestricted Fibonacci and Lucas $2^{N}$-ons. The following theorem gives the Catalan identities for unrestricted Fibonacci and Lucas $2^{N}$-ons.

Theorem 3 (Catalan identity). For any integers $r$ and $s$, we have

$$
\mathcal{F}_{N, r+s}^{\vec{c}} \mathcal{F}_{N, r-s}^{\vec{c}}-\left[\mathcal{F}_{N, r}^{\vec{c}}\right]^{2}=(-1)^{r+s+1} F_{s}\left(A_{N}^{\vec{c}} F_{s}+B_{N}^{\vec{c}} L_{s}\right)
$$

and

$$
\mathcal{L}_{N, r+s}^{\vec{c}} \mathcal{L}_{N, r-s}^{\vec{c}}-\left[\mathcal{L}_{N, r}^{\vec{c}}\right]^{2}=5(-1)^{r+s} F_{s}\left(A_{N}^{\vec{c}} F_{s}+B_{N}^{\vec{c}} L_{s}\right) .
$$

Proof. From (2) and (6) we have

$$
\begin{aligned}
& \mathcal{F}_{N, r+s}^{\vec{c}} \mathcal{F}_{N, r-s}^{\vec{c}}-\left[\mathcal{F}_{N, r}^{\vec{c}}\right]^{2} \\
& \quad=\frac{1}{5}\left[\left(\breve{\alpha} \alpha^{r+s}-\breve{\beta} \beta^{r+s}\right)\left(\breve{\alpha} \alpha^{r-s}-\breve{\beta} \beta^{r-s}\right)-\left(\breve{\alpha} \alpha^{r}-\breve{\beta} \beta^{r}\right)^{2}\right] \\
& \quad=\frac{1}{5}\left[\left((-1)^{r-s+1}\left(\breve{\alpha} \breve{\beta} \alpha^{2 s}+\breve{\beta} \breve{\alpha} \beta^{2 s}\right)+(-1)^{r} 2 A_{N}^{\vec{c}}\right] .\right.
\end{aligned}
$$


Using (4) and (5), we get

$$
\begin{aligned}
& \mathcal{F}_{N, r+s}^{\vec{c}} \mathcal{F}_{N, r-s}^{\vec{c}}-\left[\mathcal{F}_{N, r}^{\vec{c}}\right]^{2} \\
& \quad=\frac{1}{5}\left[(-1)^{r+s-1}\left(\left(A_{N}^{\vec{c}}+\sqrt{5} B_{N}^{\vec{c}}\right) \alpha^{2 s}+\left(A_{N}^{\vec{c}}-\sqrt{5} B_{N}^{\vec{c}}\right) \beta^{2 s}\right)+(-1)^{r} A_{N}^{\vec{c}}\right] \\
& \quad=\frac{1}{5}\left[(-1)^{r+s-1}\left(A_{N}^{\vec{c}}\left(\alpha^{2 s}+\beta^{2 s}\right)+5 B_{N}^{\vec{c}}\left(\frac{\alpha^{2 s}-\beta^{2 s}}{\alpha-\beta}\right)\right)+(-1)^{r} A_{N}^{\vec{c}}\right] \\
& \quad=\frac{1}{5}\left[(-1)^{r+s-1}\left(A_{N}^{\vec{c}} L_{2 s}+5 B_{N}^{\vec{c}} F_{2 s}\right)+(-1)^{r} A_{N}^{\vec{c}}\right] .
\end{aligned}
$$

The identity $5 F_{r}^{2}=L_{2 r}-(-1)^{r}$ (see $[9, \mathrm{p} .42]$ ) gives

$$
\mathcal{F}_{N, r+s}^{\vec{c}} \mathcal{F}_{N, r-s}^{\vec{c}}-\left[\mathcal{F}_{N, r}^{\vec{c}}\right]^{2}=(-1)^{r+s+1}\left(A_{N}^{\vec{c}} F_{s}^{2}+B_{N}^{\vec{c}} F_{2 s}\right),
$$

and (12) can be proved by substituting the identity $F_{2 s}=F_{s} L_{s}$ into the last equation. The Catalan identity for unrestricted Lucas $2^{N}$-ons can be obtained similarly.

If we choose $\vec{c}=(0,1,2,3)$ with $N=2$, then we obtain the respective Catalan identities for Fibonacci and Lucas quaternions:

$$
\mathcal{F}_{2, r+s}^{\vec{c}} \mathcal{F}_{2, r-s}^{\vec{c}}-\left[\mathcal{F}_{2, r}^{\vec{c}}\right]^{2}=(-1)^{r+s+1} F_{s}\left[F_{s} \mathcal{L}_{2,0}+L_{s}\left(-e_{1}-e_{2}+e_{3}\right)\right]
$$

and

$$
\mathcal{L}_{2, r+s}^{\vec{c}} \mathcal{L}_{2, r-s}^{\vec{c}}-\left[\mathcal{L}_{2, r}^{\vec{c}}\right]^{2}=5(-1)^{r+s} F_{s}\left[F_{s} \mathcal{L}_{2,0}+L_{s}\left(-e_{1}-e_{2}+e_{3}\right)\right] .
$$

The Catalan identities for the case $s=1$ become the Cassini identities for unrestricted Fibonacci and Lucas $2^{N}$-ons, and are given in the following result.

Corollary 1 (Cassini identity). For any integer $r$, we have

$$
\mathcal{F}_{N, r+1}^{\vec{c}} \mathcal{F}_{N, r-1}^{\vec{c}}-\left[\mathcal{F}_{N, r}^{\vec{c}}\right]^{2}=(-1)^{r}\left(A_{N}^{\vec{c}}+B_{N}^{\vec{c}}\right)
$$

and

$$
\mathcal{L}_{N, r+1}^{\vec{c}} \mathcal{L}_{N, r-1}^{\vec{c}}-\left[\mathcal{L}_{N, r}^{\vec{c}}\right]^{2}=-5(-1)^{r}\left(A_{N}^{\vec{c}}+B_{N}^{\vec{c}}\right) .
$$

Kecilioglu and Akkus [8] gave the Cassini identities for Fibonacci octonions. Here we obtain the Cassini identities for Fibonacci and Lucas octonions by choosing $\vec{c}=(0,1,2, \ldots, 7)$ with $N=3$ :

$$
\begin{aligned}
\mathcal{F}_{3, r+1}^{\vec{c}} \mathcal{F}_{3, r-1}^{\vec{c}}-\left[\mathcal{F}_{3, r}^{\vec{c}}\right]^{2} & =(-1)^{r}\left(\mathcal{L}_{3,0}-e_{1}-e_{2}-2 e_{3}-3 e_{4}+9 e_{5}+6 e_{6}-6 e_{7}\right) \\
& =(-1)^{r}\left(\mathcal{L}_{3,0}-\mathcal{F}_{3,0}+14 e_{5}+14 e_{6}+7 e_{7}\right)
\end{aligned}
$$


and

$$
\mathcal{L}_{3, r+1}^{\vec{c}} \mathcal{L}_{3, r-1}^{\vec{c}}-\left[\mathcal{L}_{3, r}^{\vec{c}}\right]^{2}=-5(-1)^{r}\left(\mathcal{L}_{3,0}-\mathcal{F}_{3,0}+14 e_{5}+14 e_{6}+7 e_{7}\right) .
$$

Now we present the d'Ocagne identities for unrestricted Fibonacci and Lucas $2^{N}$-ons.

Theorem 4 (d'Ocagne identity). For any integers $r$ and $s$, we have

$$
\mathcal{F}_{N, r}^{\vec{c}} \mathcal{F}_{N, s+1}^{\vec{c}}-\mathcal{F}_{N, r+1}^{\vec{c}} \mathcal{F}_{N, s}^{\vec{c}}=(-1)^{s}\left(A_{N}^{\vec{c}} F_{r-s}+B_{N}^{\vec{c}} L_{r-s}\right)
$$

and

$$
\mathcal{L}_{N, r}^{\vec{c}} \mathcal{F}_{L, s+1}^{\vec{c}}-\mathcal{L}_{N, r+1}^{\vec{c}} \mathcal{L}_{N, s}^{\vec{c}}=-5(-1)^{s}\left(A_{N}^{\vec{c}} F_{r-s}+B_{N}^{\vec{c}} L_{r-s}\right) .
$$

Proof. From Binet's formula for unrestricted Fibonacci quaternions we have

$$
\begin{aligned}
\mathcal{F}_{N, r}^{\vec{c}} \mathcal{F}_{N, s+1}^{\vec{c}}-\mathcal{F}_{N, r+1}^{\vec{c}} \mathcal{F}_{N, s}^{\vec{c}}= & \frac{1}{5}\left[\left(\breve{\alpha} \alpha^{r}-\breve{\beta} \beta^{r}\right)\left(\breve{\alpha} \alpha^{s+1}-\breve{\beta} \beta^{s+1}\right)\right. \\
& \left.-\left(\breve{\alpha} \alpha^{r+1}-\breve{\beta} \beta^{r+1}\right)\left(\breve{\alpha} \alpha^{s}-\breve{\beta} \beta^{s}\right)\right] \\
= & \frac{\sqrt{5}}{5}(-1)^{s}\left(\breve{\alpha} \breve{\beta} \alpha^{r-s}-\breve{\beta} \breve{\alpha} \beta^{r-s}\right) .
\end{aligned}
$$

Substituting (4) and (5) into the right-hand side of the last equation, we have

$$
\begin{aligned}
& \mathcal{F}_{N, r}^{\vec{c}} \mathcal{F}_{N, s+1}^{\vec{c}}-\mathcal{F}_{N, r+1}^{\vec{c}} \mathcal{F}_{N, s}^{\vec{c}} \\
& \quad=\frac{\sqrt{5}}{5}(-1)^{s}\left[\left(A_{N}^{\vec{c}}+B_{N}^{\vec{c}} \sqrt{5}\right) \alpha^{r-s}-\left(A_{N}^{\vec{c}}-B_{N}^{\vec{c}} \sqrt{5}\right) \beta^{r-s}\right] \\
& \quad=\frac{\sqrt{5}}{5}(-1)^{s}\left[A_{N}^{\vec{c}}\left(\alpha^{r-s}-\beta^{r-s}\right)+B_{N}^{\vec{c}} \sqrt{5}\left(\alpha^{r-s}+\beta^{r-s}\right)\right] .
\end{aligned}
$$

We can obtain (13) from the last equation, and (14) can be obtained in a similar way.

Choosing $\vec{c}=(0,1,2,3)$ with $N=2$, we obtain the respective d'Ocagne identities for Fibonacci and Lucas quaternions:

$$
\mathcal{F}_{N, r}^{\vec{c}} \mathcal{F}_{N, s+1}^{\vec{c}}-\mathcal{F}_{N, r+1}^{\vec{c}} \mathcal{F}_{N, s}^{\vec{c}}=(-1)^{s}\left[\mathcal{L}_{N, 0}^{\vec{c}} F_{r-s}+\left(-e_{1}-e_{2}+e_{3}\right) L_{r-s}\right]
$$

and

$$
\mathcal{L}_{N, r}^{\vec{c}} \mathcal{L}_{N, s+1}^{\vec{c}}-\mathcal{L}_{N, r+1}^{\vec{c}} \mathcal{L}_{N, s}^{\vec{c}}=-5(-1)^{s}\left[\mathcal{L}_{N, 0}^{\vec{c}} F_{r-s}+\left(-e_{1}-e_{2}+e_{3}\right) L_{r-s}\right] .
$$

Next, we present several properties for our unrestricted Fibonacci and Lucas quaternions. 
Theorem 5. For any integers $r$ and $k$, we have

$$
\mathcal{F}_{N, r+k}^{\left(c_{0}, c_{1}, \ldots, c_{2} N_{-1}\right)}=\mathcal{F}_{N, r}^{\left(c_{0}+k, c_{1}+k, \ldots, c_{2} N_{-1}+k\right)}
$$

and

$$
\mathcal{L}_{N, r+k}^{\left(c_{0}, c_{1}, \ldots, c_{2} N_{-1}\right)}=\mathcal{L}_{N, r}^{\left(c_{0}+k, c_{1}+k, \ldots, c_{2} N_{-1}+k\right)} .
$$

Proof. We only present here the proof of (15), as (16) can be proved similarly. By the definition of unrestricted Fibonacci $2^{N}$-ons, we can write

$$
\begin{aligned}
\mathcal{F}_{N, r+k}^{\left(c_{0}, c_{1}, \ldots, c_{2} N_{-1}\right)} & =F_{r+k+c_{0}} e_{0}+F_{r+k+c_{1}} e_{1}+\cdots+F_{r+k+c_{2} N_{-1}} e_{2^{N-1}} \\
& =F_{r+c_{0}+k} e_{0}+F_{r+c_{1}+k} e_{1}+\cdots+F_{r+c_{2} N_{-1}+k} e_{2^{N-1}} \\
& =\mathcal{F}_{N, r}^{\left(c_{0}+k, c_{1}+k, \ldots, c_{2} N_{-1}+k\right)} .
\end{aligned}
$$

This completes the proof.

In the next theorem, we give certain identities involving convolutions of unrestricted Fibonacci and Lucas $2^{N}$-ons.

Theorem 6. For any integers $r, s$ and $t$, we have

$$
\begin{aligned}
& \mathcal{L}_{N, r}^{\vec{c}}=\mathcal{F}_{N, r-1}^{\vec{c}}+\mathcal{F}_{N, r+1}^{\vec{c}}, \\
& \mathcal{L}_{N, r+s}^{\vec{c}} \mathcal{F}_{N, r+t}^{\vec{c}}-\mathcal{L}_{N, r+t}^{\vec{c}} \mathcal{F}_{N, r+s}^{\vec{c}}=2(-1)^{r+s} A_{N}^{\vec{c}} \mathcal{F}_{N, t-s}^{\vec{c}}, \\
& \mathcal{F}_{N, r}^{\vec{c}} \mathcal{L}_{N, s}^{\vec{c}}-\mathcal{L}_{N, s}^{\vec{c}} \mathcal{F}_{N, r}^{\vec{c}}=2(-1)^{r} B_{N}^{\vec{c}} L_{s-r}, \\
& \mathcal{F}_{N, r}^{\vec{c}} \mathcal{L}_{N, s}^{\vec{c}}-\mathcal{L}_{N, r}^{\vec{c}} \mathcal{F}_{N, s}^{\vec{c}}=2(-1)^{s}\left(A_{N}^{\vec{c}} F_{r-s}+B_{N}^{\vec{c}} L_{r-s}\right), \\
& \mathcal{F}_{N, r}^{\vec{c}} \mathcal{F}_{N, s}^{\vec{c}}-\mathcal{F}_{N, s}^{\vec{c}} \mathcal{F}_{N, r}^{\vec{c}}=2(-1)^{s+1} B_{N}^{\vec{c}} F_{r-s}, \\
& \mathcal{L}_{N, r}^{\vec{c}} \mathcal{L}_{N, s}^{\vec{c}}-\mathcal{L}_{N, s}^{\vec{c}} \mathcal{L}_{N, r}^{\vec{c}}=10(-1)^{s} B_{N}^{\vec{c}} F_{r-s}, \\
& \mathcal{F}_{N, r+s}^{\vec{c}} F_{r+s}-\mathcal{F}_{N, r-s}^{\vec{c}} F_{r-s}=\mathcal{F}_{N, 2 r}^{\vec{c}} F_{2 s}, \\
& \mathcal{L}_{N, r+s}^{\vec{c}} L_{r+s}-\mathcal{L}_{N, r-s}^{\vec{c}} L_{r-s}=5 \mathcal{F}_{N, 2 r}^{\vec{c}} F_{2 s}, \\
& \mathcal{F}_{N, r+s}^{\vec{c}} L_{r+s}-\mathcal{F}_{N, r-s}^{\vec{c}} L_{r-s}=\mathcal{F}_{N, 2 r}^{\vec{c}} L_{2 s}+2(-1)^{r+s} \mathcal{F}_{N, 0}^{\vec{c}}, \\
& \mathcal{L}_{N, r+s}^{\vec{c}} L_{r+s}-\mathcal{L}_{N, r-s}^{\vec{c}} L_{r-s}=\mathcal{L}_{N, 2 r}^{\vec{c}} L_{2 s}+2(-1)^{r+s} \mathcal{L}_{N, 0}^{\vec{c}}, \\
& 5\left[\mathcal{F}_{N, r}^{\vec{c}}\right]^{2}-\left[\mathcal{F}_{N, r}^{\vec{c}}\right]^{2}=4(-1)^{r+1} A_{N}^{\vec{c}}, \\
& \mathcal{F}_{N, r+s}^{\vec{c}}+(-1)^{n} \mathcal{F}_{N, r-s}^{\vec{c}}=\mathcal{F}_{N, r}^{\vec{c}} L_{s}, \\
& \mathcal{L}_{N, r+s}^{\vec{c}}+(-1)^{n} \mathcal{L}_{N, r-s}^{\vec{c}}=\mathcal{L}_{N, r}^{\vec{c}} L_{s}, \\
& \mathcal{F}_{N, 2 r}^{\vec{c}}=F_{r+1} \mathcal{F}_{N, r}^{\vec{c}}+F_{r} \mathcal{F}_{N, r-1}^{\vec{c}} .
\end{aligned}
$$

Proof. The above identities can be proved, for example, using a method based on the Binet's formulas for the corresponding $2^{N}$-ons. We will only 
prove (17) and (18) since the others can be proved similarly. Using Binet's formula for unrestricted Fibonacci $2^{N}$-ons, we have

$$
\begin{aligned}
\mathcal{F}_{N, r}^{\vec{c}} \mathcal{F}_{N, s}^{\vec{c}}-\mathcal{F}_{N, r}^{\vec{c}} \mathcal{F}_{N, s}^{\vec{c}}= & \frac{1}{5}\left[\left(\breve{\alpha} \alpha^{r}-\breve{\beta} \beta^{r}\right)\left(\breve{\alpha} \alpha^{s}-\breve{\beta} \beta^{s}\right)\right. \\
& \left.-\left(\breve{\alpha} \alpha^{s}-\breve{\beta} \beta^{s}\right)\left(\breve{\alpha} \alpha^{r}-\breve{\beta} \beta^{r}\right)\right] \\
= & \frac{1}{5}\left[-\breve{\alpha} \breve{\beta} \alpha^{r} \beta^{s}-\breve{\beta} \breve{\alpha} \alpha^{s} \beta^{r}+\breve{\alpha} \breve{\beta} \alpha^{s} \beta^{r}+\breve{\beta} \breve{\alpha} \alpha^{r} \beta^{s}\right] .
\end{aligned}
$$

By substituting (4) and (5) into the previous equation we get

$$
\begin{aligned}
\mathcal{F}_{N, r}^{\vec{c}} \mathcal{F}_{N, s}^{\vec{c}}-\mathcal{F}_{N, r}^{\vec{c}} \mathcal{F}_{N, s}^{\vec{c}} & =\frac{1}{5}\left[-\alpha^{r} \beta^{s} 2 \sqrt{5} B_{N}^{\vec{c}}+\alpha^{s} \beta^{r} 2 \sqrt{5} B_{N}^{\vec{c}}\right] \\
& =-\frac{2 \sqrt{5}}{5} B_{N}^{\vec{c}} \alpha^{s} \beta^{s}\left(\alpha^{r-s}-\beta^{r-s}\right) \\
& =2(-1)^{s+1} B_{N}^{\vec{c}} F_{r-s} .
\end{aligned}
$$

Similarly, using Binet's formula again, we obtain

$$
\begin{aligned}
\mathcal{L}_{N, r+s}^{\vec{c}} L_{r+s}-\mathcal{L}_{N, r-s}^{\vec{c}} L_{r-s}= & \left(\breve{\alpha} \alpha^{r+s}+\breve{\beta} \beta^{r+s}\right)\left(\alpha^{r+s}+\beta^{r+s}\right) \\
& -\left(\breve{\alpha} \alpha^{r-s}+\breve{\beta} \beta^{r-s}\right)\left(\alpha^{r-s}+\beta^{r-s}\right) \\
= & \left(\breve{\alpha} \alpha^{2 r+2 s}+\breve{\beta} \beta^{2 r+2 s}-\breve{\alpha} \alpha^{2 r-2 s}-\breve{\beta} \beta^{2 r-2 s}\right) \\
= & \left(\breve{\alpha} \alpha^{2 r+2 s}+\breve{\beta} \beta^{2 r+2 s}-\breve{\alpha} \alpha^{2 r} \beta^{2 s}-\breve{\beta} \alpha^{2 s} \beta^{2 r}\right) \\
= & (\alpha-\beta)^{2}\left(\frac{\breve{\alpha} \alpha^{2 r}-\breve{\beta} \beta^{2 r}}{\alpha-\beta}\right)\left(\frac{\alpha^{2 s}-\beta^{2 s}}{\alpha-\beta}\right) \\
= & 5 \mathcal{F}_{N, 2 r}^{\vec{c}} F_{2 s} .
\end{aligned}
$$

The next theorem gives certain summation formulas for unrestricted Fibonacci and Lucas $2^{N}$-ons without proof, because the proofs can be obtained straightforwardly using only elementary operations.

Theorem 7. We have

$$
\begin{gathered}
\sum_{t=0}^{r} \mathcal{F}_{N, t}^{\vec{c}}=\mathcal{F}_{N, r+2}^{\vec{c}}-\mathcal{F}_{N, 1}^{\vec{c}}, \quad \sum_{t=0}^{r} \mathcal{L}_{N, t}^{\vec{c}}=\mathcal{L}_{N, r+2}^{\vec{c}}-\mathcal{L}_{N, 1}^{\vec{c}}, \\
\sum_{t=0}^{r} \mathcal{F}_{N, 2 t-1}^{\vec{c}}=\mathcal{F}_{N, 2 r}^{\vec{c}}-\mathcal{F}_{N, 0}^{\vec{c}}, \quad \sum_{t=0}^{r} \mathcal{L}_{N, 2 t-1}^{\vec{c}}=\mathcal{L}_{N, 2 r}^{\vec{c}}-\mathcal{L}_{N, 0}^{\vec{c}}, \\
\sum_{t=0}^{r} \mathcal{F}_{N, 2 t}^{\vec{c}}=\mathcal{F}_{N, 2 r+1}^{\vec{c}}-\mathcal{F}_{N,-1}^{\vec{c}}, \quad \sum_{t=0}^{r} \mathcal{L}_{N, 2 t}^{\vec{c}}=\mathcal{L}_{N, 2 r+1}^{\vec{c}}-\mathcal{L}_{N,-1}^{\vec{c}},
\end{gathered}
$$


and

$$
\sum_{t=0}^{r}\left(\begin{array}{l}
r \\
t
\end{array}\right) \mathcal{F}_{N, t}^{\vec{c}}=\mathcal{F}_{N, 2 r}^{\vec{c}}, \quad \sum_{t=0}^{r}\left(\begin{array}{l}
r \\
t
\end{array}\right) \mathcal{L}_{N, t}^{\vec{c}}=\mathcal{L}_{N, 2 r}^{\vec{c}} .
$$

\section{Acknowledgement}

This research is financed by Kastamonu University Scientific Research Projects Coordination Unit (KUBAP-01/2017-01).

\section{References}

[1] M. Akyigit, H. H. Kosal, and M. Tosun, Fibonacci generalized quaternions, Adv. Appl. Clifford Algebr. 24 (2014), 631-641.

[2] G. Bilgici, U. Tokeser, and Z. Unal, Fibonacci and Lucas sedenions, J. Integer Seq. 20 (2017), 1-11.

[3] R. E. Cawagas, On the structure and zero divisors of the Cayley-Dickson sedenion algebra, Discuss. Math. Gen. Algebra Appl. 24 (2004), 251-265.

[4] C. Flaut and V. Shpakivskyi, On generalized Fibonacci quaternions and FibonacciNarayana quaternions, Adv. Appl. Clifford Algebr. 23 (2013), 673-688.

[5] S. Halici, On Fibonacci quaternions, Adv. Appl. Clifford Algebr. 22(2) (2012), 321327.

[6] A. F. Horadam, Complex Fibonacci numbers and Fibonacci quaternions, Amer. Math. Monthly 70(3) (1963), 289-291.

[7] M. R. Iyer, A note on Fibonacci quaternions, Fibonacci Quart. 7(3) (1969), 225-229.

[8] O. Kecilioglu and I. Akkus, The Fibonacci octonions, Adv. Appl. Clifford Algebr. 25(1) (2015), 151-158.

[9] T. Koshy, Fibonacci and Lucas Numbers with Applications, Wiley, New York, 2001.

[10] M. N. S. Swamy, On generalized Fibonacci quaternions, Fibonacci Quart. 11(5) (1973), 547-550.

Department of Computer Education and Instructional Technologies, Kastamonu University, 37200 Kastamonu, Turkey

E-mail address: gbilgici@kastamonu.edu.tr

Department of Mathematics, Faculty of Arts and Sciences, Kastamonu University, 37150 Kastamonu, TURKEy

E-mail address: ahmetdasdemir37@gmail.com 